\title{
Design and study of DC excitation power controller of superconductor fault current limiter
}

\author{
Hui Li \\ Tianjin University of Technology and Education, Tianjin 300222, China \\ E-mail: lihui2957@sina.com
}

Received 13 September 2017; accepted 20 September 2017

DOI https://doi.org/10.21595/vp.2017.19173

Check for updates

\begin{abstract}
This paper provides a theoretical basis for high voltage magnetization and low voltage constant current excitation. The design structure of DC excitation power supply is put forward. As a controlled rectifier device, the 6RA70 is the core control unit of the superconducting fault current limiter. The problem that DC excitation power supply is difficult to control under high voltage and large current is effectively solved. The requirements of high voltage magnetization and constant current excitation control are expounded, and the switching methods between two states are described. PLC is used to establish a digital control system for networks. This paper provides a reference for the operation of superconducting fault current limiter.
\end{abstract}

Keywords: superconducting fault current limiter, DC excitation power supply, control network.

\section{Introduction}

With the expansion of power system installed capacity and rapid increase of grid connected power stations, short-circuit faults will inevitably occur in power grid, and the rise of short-circuit current will seriously threaten the safety of power grid operation. This would lead to high electric currents, hence threaten the safe operations of the grids. A traditional way to limit the currents is to cascade large reactance to the grids. This may be effective, but it would bring unfavorable factors such as great energy-wasting and safety issues under normal operations [1]. A saturated iron-core superconducting fault current limiter (SCFCL) with controllable DC excitation power supply can effectively solve those problem. This current limiter provides excitation current for superconducting winding by using controllable DC power supply, which makes the AC reactance in deep saturated states cascaded to power grid and the impedance of them close to zero. Once short-circuits happen, the reactors will exit the deep saturated states and remain high-impedance. In this way, current will be sufficiently limited [2], and the reclosing requirements will be automatically ensured. For this purpose, it is pivotal to design a stable DC excitation power supply and a reliable control system in the safe operation of the superconducting fault current limiter.

\section{The working principle of the superconducting fault current limiter}

The structure of an SCFCL is depicted in Fig. 1. An SCFCL consists of the iron-core of the current limiter, DC superconducting winding, AC winding, and DC control circuit.

When the current limiter is under operation, the exciting current in the superconducting winding, which is provided by the DC power supply, makes the iron-core in deep saturated state. The AC side winding exhibits a low impedance at this time. When the current of the grid in the $\mathrm{AC}$ winding does not exceed the rated value, the grid runs in the normal state of no current limit. If short-circuit fault happens, the DC excitation power supply will be immediately cut off, and the iron-core of the current limiter will leave the saturated state. At the same time, as the current caused by the short-circuit increases, the impedance of the AC winding increases immediately, which will confine the increasing of the short-circuit current. Therefore, the inductance blocking current plays a decisive role in limiting the grid current to rise sharply after exceeding the rated current.

The controllable DC winding is made of superconducting materials, which make the resistance of it almost zero in superconducting state, mainly to avoid the resistance loss caused by the coil 
of DC magnetizing winding. In addition, superconducting materials can afford higher current density than normal conductors, and with the same winding parameters, superconducting materials can provide greater DC magnetic field [3, 4].

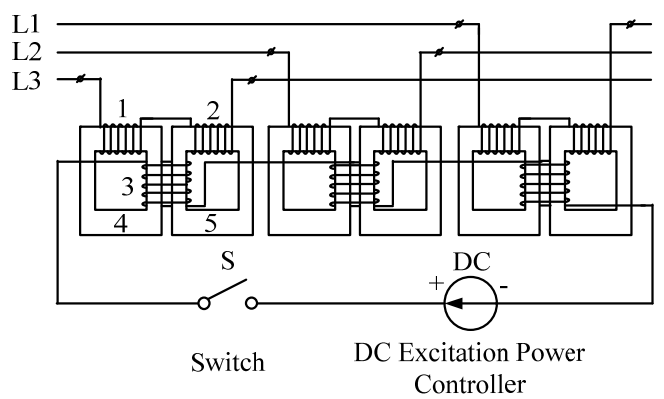

Fig. 1. Structure of SCFCL_1, 2- AC winding;

3 - DC superconducting winding; 4,5 - iron-core of SCFCL

\section{Design and study of the DC controllable power supply}

\subsection{Study of the excitation process}

The role of the DC power supply is not only to provide exciting current but also to meet the controlling requirements of the operation of the power grid and those of the response of short circuit. At the same time, the efficiency of the long-term operation of the grid should also be considered. The relay protection of the power grid requires the grid to reclose within $1 \mathrm{~s}$ after short-circuit, and requires that the DC excitation process should be completed before reclosing. The DC winding of the SCFCL is a superconducting winding with an iron-core, which is saturable and made of soft magnetic materials. Thus, it is essentially a nonlinear inductor with an iron-core. The excitation process of the saturable core can be analyzed by a piecewise linearized $\Psi$-i curve, as shown in Fig. 2.

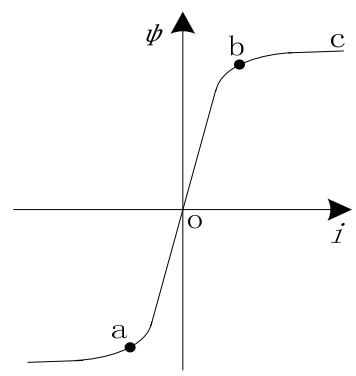

Fig. 2. Piecewise Linearized $\Psi$-i Curve of the saturableiron-core

As in Fig. 2, the $\Psi$-i curve consists of three pieces. In the middle segment (a-b), the iron-core is not saturated. As the current increases, the magnetic flow increases significantly, and the inductance value is big. In the other two segments, as the current changes, the magnetic flow changes insignificantly, and the inductance value is close to zero. Because the purpose of the dc excitation is to saturate the core as quickly as possible, the excitation process consists of two segments, unsaturated segments (o-b) and deep saturated segments (b-c). When the iron-core is not saturated, in order to increase the current of the inductance to a higher value, high voltage needs to be added to the inductance. Therefore, step high constant voltage is adopted to magnetize the core until it is saturated. When the iron-core is deep saturated, the inductance is almost zero. Because the coil is winded by superconducting materials, the resistance is also close to zero. If 
one magnetizes the core with high voltage, the current will increase rapidly, which will lead to huge power consumption and burn the winding. Hence, Therefore, it is more effective to provide constant current by means of low pressure constant current excitation. It can not only meet the requirements of deep saturated superconducting winding core, but also ensure the safety of windings and improve the efficiency of power supply at runtime.

\subsection{Parameter determination of the superconducting limiter's excitation process}

According to the study of the excitation process, the excitation includes two stages, the high-voltage magnetization stages and the low-voltage constant current excitation stages. The parameters of the excitation process of the superconducting limiter are shown in Table 1. The values of the parameters in this table are only designed for this paper. In practice, the controlling parameters should be adjusted according to the particular SCFCL.

Table 1. Controlling parameters

\begin{tabular}{|c|c|c|c|}
\hline & Current & Response time & Maximum inductance \\
\hline Iron-core saturation & $50 \mathrm{~A}$ & $\leq 300 \mathrm{~ms}$ & \multirow{2}{*}{$2 \mathrm{H}$} \\
\hline Iron-core depth saturation & $200-250 \mathrm{~A}$ & $\leq 200 \mathrm{~ms}$ & \\
\hline
\end{tabular}

According to Table 1, the high-voltage magnetization current should increase to $50 \mathrm{~A}$ in $300 \mathrm{~ms}$. After the core saturation, in order to prevent the current from increasing rapidly and burning the winding, current-limiting resistance must be added to the circuit. In addition, the resistance should be able to ensure that the current will never exceed $250 \mathrm{~A}$ under the highest possible voltage, otherwise the time of magnetization would be affected. Therefore, the resistance is determined to be $10 \Omega$.

When the inductance is $2 \mathrm{H}$ and the resistance is $10 \Omega$, according to the zero-state response of First Order Circuit, the requirement of $300 \mathrm{~ms}$ response time can be satisfied when the voltage is $644 \mathrm{~V}$ and the high-voltage magnetization current is 50A. After the saturation, the constant current excitation process proceeds in a winding which is an approximate non impedance conductor. Hence, the constant current excitation time (as the current increases from $50 \mathrm{~A}$ to $250 \mathrm{~A}$ ) is almost zero, as shown in Table 2.

Table 2. The response time of high-voltage magnetization and constant current excitation

\begin{tabular}{|c|c|c|}
\hline \multirow{2}{*}{ Voltage (V) } & \multicolumn{2}{|c|}{ Time (ms) } \\
\cline { 2 - 3 } & High-voltage magnetization & Constant current excitation \\
\hline 644 & 300 & 0.1 \\
\hline 1000 & 139 & 0.11 \\
\hline
\end{tabular}

The operation response time of the system is mainly determined by the high-voltage magnetization time. According to Table 2, the $1 \mathrm{~s}$ reclosing requirement can be satisfied. As the voltage of magnetization increases, the time of magnetization decreases. When the core is deep saturated, the current is the constant excitation current, which should be a stable value between $200 \mathrm{~A}$ and $250 \mathrm{~A}$.

\subsection{Design of the DC excitation power supply controller}

The rectifying devices nowadays are very mature $[5,6]$. Developing a rectifier with large capacity and good controllability will affect the design cycle of the system. The suitable rectifier device is selected directly, which can meet the control requirements.

Siemens 6RA70 series DC speed regulating device is essentially a high-performance controllable rectifying device. It mainly provides controllable and stable DC voltage and current for DC motors. Since the DC superconducting winding and the winding of DC motor both have inductive load, the same technique can be used to provide power and to control superconducting 
DC winding. 6RA70 can output 15-3000 A DC current and $1300 \mathrm{~V}$ DC voltage, which just meets the requirements of high voltage and large current in the superconducting DC excitation process. In addition, 6RA70 has good controllability and communication, and can realize voltage closed loop and current closed loop output by changing the parameters. Therefore, the problem of high voltage and current in the DC power supply can be solved by using 6RA70 as the core component of the controllable DC power supply. The controlling requirements under operation can also be solved. Please refer to Fig. 3 for the structure of the Controllable DC power supply.

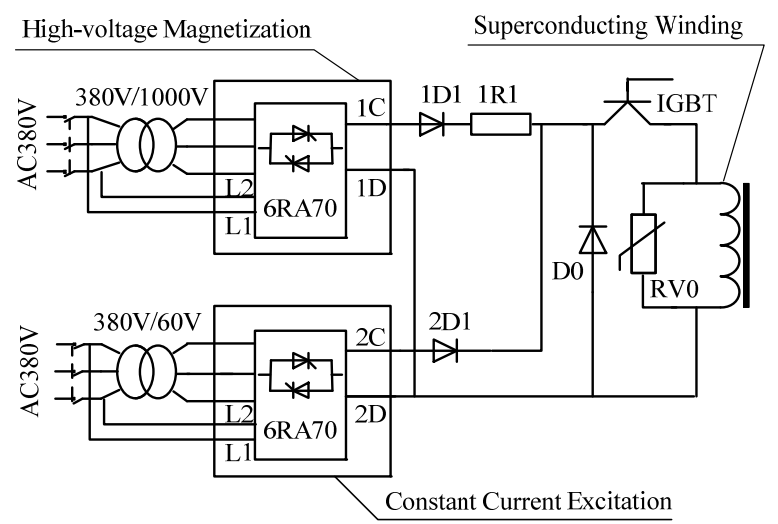

Fig. 3. Structure of the controllable DC power supply

\subsection{High voltage magnetization}

High voltage magnetizing is a forced high voltage in the range of $644-1000 \mathrm{~V}$ at both ends of the DC superconducting winding, so that the current in the winding rises rapidly. The specific voltage value can be set according to the actual situation. The system uses step-up transformer to provide an input voltage of about $1000 \mathrm{~V}$. The output terminal of the armature winding of the 6RA70 is connected to the DC superconducting winding and is set to the voltage closed loop output mode. As the high-voltage magnetized 6RA70 outputs constant high voltage, the current in the superconducting winding increases rapidly. When the current reaches $50 \mathrm{~A}$, the iron-core tends to be saturated. After the winding lost their inductance, the current will increase almost perpendicularly. At that time, it is not necessary to operate under high voltage mode. It should be turned to constant current excitation.

\subsection{Constant current excitation}

In order to deep saturate the core of the superconducting current limiter, the working current of the DC superconducting winding should be maintained within 200-250 A. As the impedance reaches zero, a stable current of $250 \mathrm{~A}$ can be produced by relatively low voltage. If we adopt low voltage and high current, the efficiency of the power supply can be improved, and the requirement of large current can also be achieved. Though the reactance of the superconducting winding is zero, in practice, the electric corrosion of the circuit and the contact resistance of the device must be considered. In electrical applications, empirically, this resistance is limited within $0.2 \Omega$, and the output of the low-voltage constant-current excitation is assumed to be $60 \mathrm{~V}$. The system provide a voltage for the constant current excited 6RA70 using the step-down transformer. It connects the armature output end of 6RA70 to the DC superconducting winding and set it to the closed-loop current mode in which the current output within 200-250 A. When the high-voltage magnetization increases the current to $50 \mathrm{~A}$, rapidly switch the limiter to constant-current excitation. The core of the limiter would be deep saturated and operation stably. 


\subsection{Switch between the high-voltage magnetization current and the constant-current excitation current}

Because of mechanical contact contactor action is slow, cannot satisfy under "seconds" level for effective control, also can produce arc and the phenomenon of blocks the flow, so the magnetization current and high voltage constant current for magnetic current switch adopts non-contact switch (i.e., power diode) [7,8]. To complete the system inputs high and low voltages in parallel, and uses power diode to isolate them. In high-voltage magnetization, the current starts from 1C, via 1D1, 1R1, IGBT, and the superconducting winding, returns along 1D, and 2D1 is reverse cut off. In constant current excitation, the current starts from $2 \mathrm{C}$, via $2 \mathrm{D} 1$, IGBT, and the superconducting winding, returns along 2D, and 1D1 is reverse cut off. Therefore, the mechanical contact switch can be omitted, and the shortcoming of slow action can be avoided. The switching between high-voltage magnetization and constant current excitation is accomplished by PLC transmitting control signal through Profibus-DP.

\section{Control network}

\subsection{Network structure}

The control system uses Siemens S7-300 PLC as the master station. Two 6RA70rectification control device is used as high-voltage magnetization and constant current excitation slave stations, respectively. Through the touch screen, the human-computer interface and the digital networking control are established. The structure of the network is shown in Fig. 4.

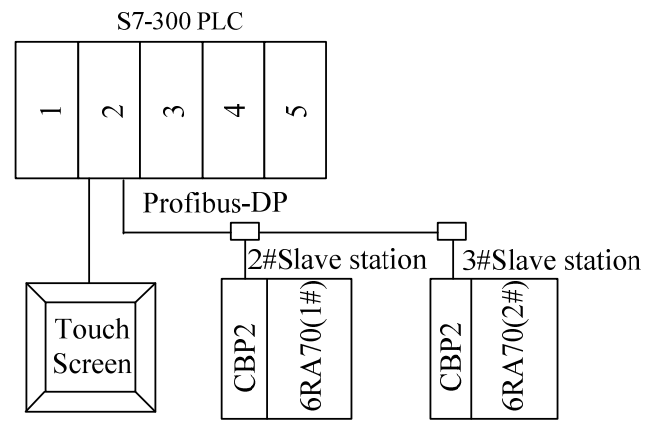

Fig. 4. Structure of the network: 1 - power supply module; 2 - CPU module; 3 - analog input module; 4 -digital input module; 5 - digital output module

\subsection{System operation process}

The start and the stop signals of the DC excitation power supply come from the short circuit control device of the power grid. In order to reduce the switching time between high-voltage magnetization and constant current excitation, the operation process of 6RA70 consists of two procedures, the start-up procedure and the enabling procedure. In the star-up procedure, the device is initialized, and incoming contactor of 6RA70 switches on. In the enabling procedure, a trigger pulse is transmitted to the thyristor. After these two procedures, 6RA70 is able to produce output. When PLC receives the start signal, it transmits the start-up control word and the enabling control word to the No. 2 slave station 6RA70 (1\#) through Profibus-DP, at the same time transmits start-up control word to No. 3 slave station 6RA70 (2\#). Thus, 6RA70 (1\#) outputs DC high voltage by means of voltage closed loop, which makes the current in the superconducting DC winding increase rapidly, and 6RA70 (2\#) waits for the enabling signal. When the current reaches 50 A, PLC transmits stop control word to the high-voltage magnetized 6RA70 (1\#) to halt the magnetization process, and transmits enabling control word to 6RA70 (2\#) for constant current 
excited. At this point, 6RA70 (2\#) outputs by means of current closed loop, which makes the current rapidly increase to the preset values and stably maintain at it. When the short circuit fault occurs to the grid, the monitoring device of short circuit current transmits off signals to IGBT and PLC at the same time. IGBT turns off immediately. The DC excitation power supply of the superconducting winding of the limiter is cut off, and the back EMF generated by the DC superconducting winding is released by the RV0 piezoresistor. PLC then transmits enabling-prohibiting signal. 6RA70 stops outputting and waits for reclosing signal.

Under operation, the system sets the voltage, the current, and the start and stop control words of 6RA70 through the touch screen. They are then transmitted to 6RA70 by PLC through Profibus-DP. Finally, the operation status signal, the fault signal, and the actual voltage and current values are delivered back to PLC via Profibus-DP in the form of status word, and shown in the touch screen.

\section{Conclusions}

First, the system uses the voltage closed loop output of 6RA70 as the high-voltage magnetization DC excitation power supply. The impact of the inductance of the superconducting winding on the excitation time is solved by means of high voltage. The requirement of fast excitation is also satisfied. Secondly, the excitation is achieved by 6RA70 current closed loop outputting low voltage constant current, which guarantees the safety of the superconducting winding and enhances the efficiency of the power supply. Thirdly, the switching time between the high-voltage magnetization current and the constant current excitation current is decreased by the non-contact switch. In addition, the control is achieved by the network of Profibus-DP through S7-300 PLC, which prevents complex wiring and improves the system's stability and resistant to interference. Finally, the adjustability of the voltage and the current enhances the flexibility of the system. Nowadays, as the expansion of power system and industrialization of superconducting fault restrictor, this design has important practical significance and reference value.

\section{References}

[1] Xiao Liye, Lin Liangzhen Superconductivity FCL - a leading product of industrialization of superconductivity technology. Science and Technology Review, Vol. 7, 1999, p. 37-44.

[2] Zhang Xuhong, Zhou Youqing The configurations and characteristics of the superconducting fault current limiters. Cryogenics and Superconductivity, Vol. 4, 2002, p. 22-23.

[3] Raju B. P. A current limiting device using SC DC bias. IEEE Transactions on Power Apparatus and Systems, Vol. 101, Issue 9, 1982, p. 3173-3177.

[4] Gong Weizhi, Zhang Jingyin, Cao Zhengjian, et al. HTS DCBias coil for $35 \mathrm{kV} / 90 \mathrm{MVA}$ saturated iron-core fault current limiter. Physica C, Vol. 468, 2007, p. 2050-2053.

[5] Nikulshin Y., Wolfus Y., Friedman A., Yeshurun Y., Rozenshtein V., Landwer D., Garbi U. Saturated core fault current limiters in a live grid. IEEE Transactions on Applied Superconductivity, Vol. 26, Issue 3, 2016, p. 5601504.

[6] Tixador P., Badel A. Superconducting fault current limiter optimized design. Physica C: Superconductivity and its Applications, Vol. 518, 2015, p. 130-133.

[7] Xin Ying, Gong Weizhi, Gao Yongquan, et al. Introduction of 35KV/90MVA saturated iron-core superconducting fault current limiter. Rare Metal Materials and Engineering, Vol. 36, Issue 3, 2007, p. 1-6.

[8] Cui Ji Bin, Sun Yu Wei, Hong Hui, et al. Study on field suppression unit in DC excitation system for saturated iron-core super conducting fault current limiter. IEEE Transactions on Applied Superconductivity, Vol. 24, Issue 5, 2014, p. 5602304. 